OPEN ACCESS

Edited by:

Matteo Bologna,

Sapienza University of Rome, Italy

Reviewed by:

Hyungmin Kim,

Korea Institute of Science and

Technology (KIST), South Korea

Jérôme Sallet,

University of Oxford, United Kingdom

*Correspondence:

Lazzaro di Biase lazzaro.dibiase@gmail.com

I.dibiase@unicampus.it

Specialty section:

This article was submitted to

Movement Disorders,

a section of the journal

Frontiers in Neurology

Received: 18 February 2019

Accepted: 07 May 2019

Published: 11 June 2019

Citation:

di Biase L, Falato E and Di Lazzaro V

(2019) Transcranial Focused

Ultrasound (tFUS) and Transcranial

Unfocused Ultrasound (tUS)

Neuromodulation: From Theoretical

Principles to Stimulation Practices.

Front. Neurol. 10:549

doi: 10.3389/fneur.2019.00549

\section{Transcranial Focused Ultrasound (tFUS) and Transcranial Unfocused Ultrasound (tUS) Neuromodulation: From Theoretical Principles to Stimulation Practices}

\author{
Lazzaro di Biase $^{1,2 *}$, Emma Falato ${ }^{1,2}$ and Vincenzo Di Lazzaro ${ }^{1}$ \\ ${ }^{1}$ Neurology, Neurophysiology, and Neurobiology Unit, Department of Medicine, Campus Bio-Medico University of Rome, \\ Rome, Italy, ${ }^{2}$ Unit of Neurophysiology and Neuroengineering of Human-Technology Interaction, School of Medicine, Campus \\ Bio-Medico University of Rome, Rome, Italy
}

Transcranial focused ultrasound is an emerging technique for non-invasive neurostimulation. Compared to magnetic or electric non-invasive brain stimulation, this technique has a higher spatial resolution and can reach deep structures. In addition, both animal and human studies suggest that, potentially, different sites of the central and peripheral nervous system can be targeted by this technique. Depending on stimulation parameters, transcranial focused ultrasound is able to determine a wide spectrum of effects, ranging from suppression or facilitation of neural activity to tissue ablation. The aim is to review the state of the art of the human transcranial focused ultrasound neuromodulation literature, including the theoretical principles which underlie the explanation of the bioeffects on neural tissues, and showing the stimulation techniques and parameters used and their outcomes in terms of clinical, neurophysiological or neuroimaging results and safety.

Keywords: focused ultrasound, transcranial stimulation, non-invasive brain stimulation (NIBS), transcranial focused ultrasound (tFUS), transcranial ultrasound (tUS)

\section{INTRODUCTION}

Preliminary animal studies suggest that, potentially, different sites in the peripheral nervous system, from nerves (1) to spinal roots (2), and in the central nervous system, from superficial regions like primary motor cortex (3) or frontal eye field (4), to more deep areas like hippocampus (3), amygdala (5), or thalamus (6) can be targeted by focused ultrasound stimulation technique. In addition, animal studies showed that this technique has a high spatial resolution, useful also for mapping small brain areas, as shown by Fry (7) for the mapping of lateral geniculate nucleus, or by Ballantine et al. (2) for the stimulation of Edinger-Westphal nucleus.

Furthermore, a recent fMRI resting-state functional connectivity animal study (8), showed that the effect of tFUS neuromodulation can last for up to $2 \mathrm{~h}$ after stimulation, opening a new way to explore not only the online effect but also the long lasting effect of neuromodulation. The first human transcranial application of ultrasounds for neuromodulation was described by Hameroff et al. (9), with an unfocused transcranial ultrasound (tUS) continuous stimulation of posterior frontal cortex, applied on 31 patients affected by chronic pain. The first human application of focused transcranial ultrasound (tFUS) technique was described by Legon et al. (10). They targeted 
the primary somatosensory cortex of healthy volunteers, in a within-subjects, sham-controlled study. One of the most interesting results of tFUS applications was a case report of emergence from minimally conscious state, after low intensity non-invasive ultrasonic thalamic stimulation in a patient after acute brain injury (11). Following this first single evidence, a clinical trial is ongoing to explore the effect of thalamic low intensity focused ultrasound in acute brain injury patients (12).

Regarding peripheral nervous system neuromodulation, Bailey et al. (13) explored the ability of continuous US at 1.5 $\mathrm{MHz}$ in modulating the ulnar nerve stimulation response to magnetic stimulation (MS). This study showed no significant change in electromyographic response during magnetic plus US ulnar nerve stimulation. However, further studies are needed in order to explore different parameter of stimulation.

In recent years, the scientific community showed a progressive increasing interest on FUS neuromodulation, and some reviews have been published in order to summarize the state of the art on this topic (14-18).

\section{Mechanisms of Actions of US Neuromodulation}

Focused ultrasound is a non-invasive, non-ionizing technique. In order to target a brain region, the first challenge is to let ultrasounds single waves to reach the target at the same time, without different acoustic reflection, refraction, and distortion due to the inhomogeneity of skull bone. This problem can be solved by time shifting each single ultrasound wave, according to the related skull bone acoustical properties, in order to let all the waves to reach the target at the same time (19-22).

The mechanical interaction between US and neuronal membranes can modify the membrane gating kinetics through the action on mechanosensitive voltage-gated ion channels or neurotransmitter receptors (23-25). The study of Tyler et al. (25) supports this hypothesis. Their study showed, on ex vivo mouse brains and hippocampal slice cultures, that low-intensity, lowfrequency ultrasound (LILFU) is able to activate voltage-gated sodium and calcium channels. However, this can't be the only mechanism of action, explaining the action potential induction, since in simulations, considering the role of membrane tension on activation of mechanically sensitive voltage gated channels, the resulting effect was too low to induce an excitation $(26,27)$.

In addition, the mechanical action of US is able to induce cavitation into the cellular membrane, by means of membrane pore formation, which changes the membrane permeability.

The bilayer sonophore model (28) was introduced to better explain the bioeffects of US, taking into consideration the biomechanical proprieties of US and of cell membranes. According to this model (28), the mechanical energy of US leads to periodic expansions and contractions of the membrane. In this model, the US bioeffect is dependent on the tension applied to the membrane. With a progressive increase in membrane stretch intensity, the bioeffect is mediated by different mechanisms. First by the activation of mechanosensitive proteins. Then, with an increase of intensity, there is a pore formation and with the maximum stretch that can be achieved with the technique a membrane rupture and irreversible lesion is obtained (28) (Figure 1).

Considering the electrical properties of the cell membrane at rest, which can be approximated with a parallel plate capacitor, a hypothesis is that the dynamic fluctuation of the membrane bilayer changes the instantaneous membrane capacitance and leads to a capacitive current, which can potentially activate voltage-dependent sodium and potassium channels (27) (Figure 2). The neuronal bilayer sonophore model (27) combines, in a complementary way, all the biomechanical and bioelectrical proprieties of the cell membrane described, and predicts the stimulation parameter needed to reach a successful motor cortex stimulation. It explains, for example, the higher efficacy of long US stimulation pulses (3, 29, 30), and how the action potential can be elicited after the end of the US stimulus $(27,31)$, with a good overlap with the experimental results obtained using real stimulation on the mouse motor cortex (30).

\section{Stimulation Parameters}

An acoustic wave can be defined by two fundamental parameters: the intensity, defined as the amplitude of the wave, and the instantaneous period $(\mathrm{T})$, defined as the time needed to complete
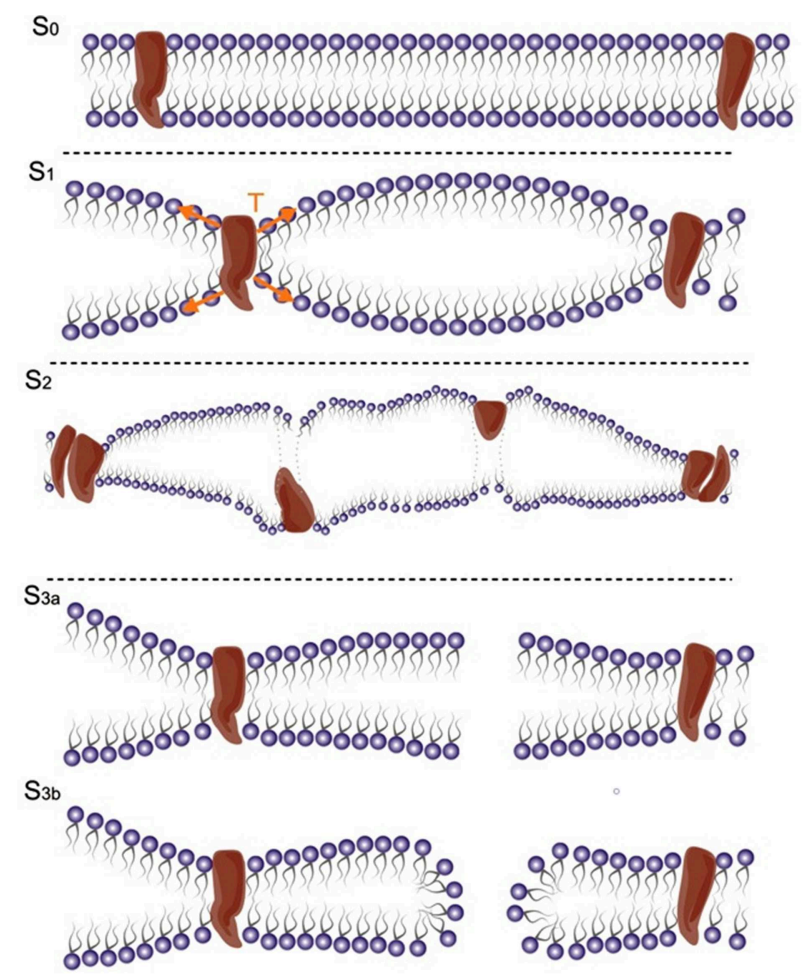

FIGURE 1 | Ultrasound gradually increases tension in the membrane. From the reference stage (SO), the stretch first activates mechanosensitive proteins (S1); growing tension might damage membrane proteins (S2) and then might induce pore formation (S3a, S3b) or cause membrane rupture [modified, with permission, from Krasovitski et al. (28)]. 
A

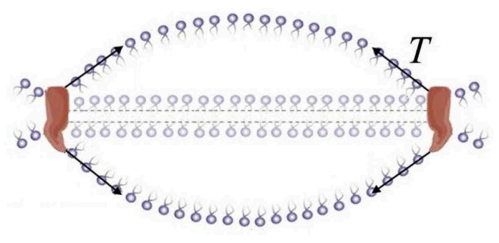

B

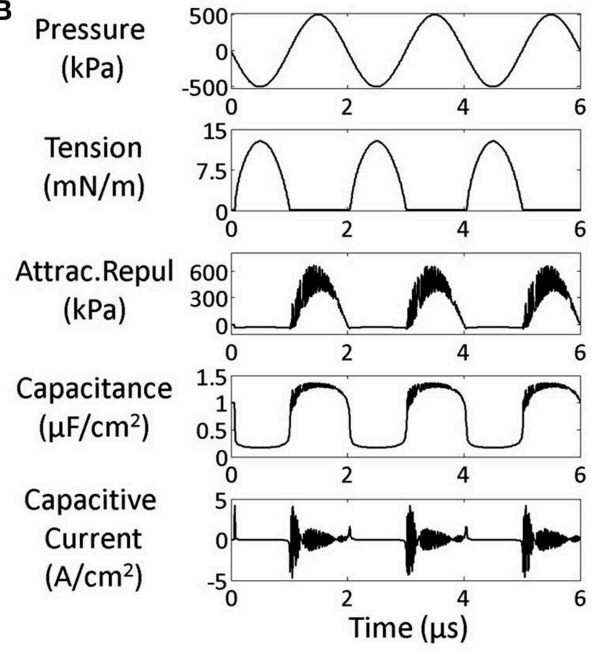

FIGURE 2 | (A) Under US effect the membrane starts fluctuating around a steady state. (B) Mechano-electrical dynamics of the model membrane to US (pressure amplitude $500 \mathrm{kPa}$ and frequency $0.5 \mathrm{MHz}$ ): The increase in Acoustic pressure induces an increase in attraction/repulsion force, which increases the capacitance leading finally to a capacitive current. Acoustic pressure $(\mathrm{kPa})$, tension $(\mathrm{mN} / \mathrm{m})$, combined attraction/repulsion force per area between the leaflets (sum of molecular and electrostatic forces, $\mathrm{kPa})$, membrane capacitance $\left(\mu \mathrm{F} / \mathrm{cm}^{2}\right)$, and capacitive displacement current $\left(\mathrm{A} / \mathrm{cm}^{2}\right)[\mathrm{modified}$, under the terms of the Creative Commons Attribution 3.0 License, from Plaksin et al. (27)].

one single oscillation cycle, which is used to calculate the Acoustic frequency (Af) (Figure 3, Equation 1). In addition to these two parameters, the stimulus duration (StimD) is the total duration of one single sonication.

During the stimulus duration two paradigms of sonication are used: continuous or pulsed. Some of these protocols resemble those used for non-invasive brain stimulation based on repetitive transcranial magnetic stimulation [see Di Lazzaro and Rothwell (32) for a review]. The most used one for neuromodulation is the pulsed paradigm.

For the pulsed paradigm, two additional periods need to be defined: the pulse duration (PD), which is the period of acoustic sonication from the starting point of oscillation to the ending point, before the pause and the pulse repetition period (PRP), which is the period between the starting point of two consecutive sonications, or, in other terms, the sum of the pulse duration (PD) and the pause between two consecutive sonications. This period is used to calculate the pulse repetition frequency (PRF) (Figure 3, Equation 2). For the pulsed paradigm, the duty cycle (DC) (Figure 3, Equation 3) is the fraction of the pulsed repetition period (PRP) covered by the pulse duration (PD). The cycles per pulse (c/p) are the number of cycles during a single pulse (Figure 3, Equation 4); instead, the number of pulses $(\mathrm{Np})$ is the number of pulses throughout the stimulus duration (Figure 3, Equation 5).

The sonication delivered during the stimulus duration period can be repeated, without pauses, for the continuous stimulation protocol. Instead, intermittent protocols are characterized by pauses between the sonications, defined as inter stimulation intervals (ISIs). The intermittent protocol is the most used for FUS neurostimulation, instead the continuous one is the most used for the unfocused stimulation (Table 1).
For safety reasons the indexes that describe the thermal and biomechanical effects of the sonication need to be defined. These parameters are related to the instantaneous intensity of stimulation and its instantaneous acoustic pressure. The two main mechanisms that can induce tissue damage are: local heating, which through proteins denaturation leads to cell death, and inertial cavitation. The latter is thought to be mediated by the collapse of gas bubbles due to the pressure exerted by ultrasonic field sufficiently strong to allow tissue damage.

Both, animal histological studies $(8,41,42)$ and human neuroimaging studies $(37,38)$, showed that it is possible to neuromodulate brain circuits without inducing tissue damage. The thermal index (TI) is the ratio of total acoustic power to the acoustic power required to raise tissue temperature by $1^{\circ} \mathrm{C}$ under defined assumptions. Finally, the non-thermal, mechanical bioeffect is described by the mechanical index (MI), which is directly proportional to the ultrasound beam's peak negative pressure and inversely proportional to the frequency of the beam.

The intensity, spatial-peak pulse-average $\left(\mathrm{I}_{\text {SPPA }}\right)$ is the value of the pulse-average intensity at the point in the acoustic field where the pulse-average intensity is a maximum or is a local maximum within a specified region. The intensity, spatial-peak temporal-average (ISPTA) is the value of the temporal-average intensity at the point in the acoustic field where the temporalaverage intensity is a maximum, or is a local maximum within a specified region.

The FDA guidelines defined the safety threshold for diagnostic usage of US for adult cephalic ultrasound, which can be applied to neuromodulation. These parameters are Isspa $\leq 190 \mathrm{~W} / \mathrm{cm}^{2}$, Ispta $\leq 94 \mathrm{~mW} / \mathrm{cm}^{2}$ and a mechanical index $\leq 1.9(43)$. 


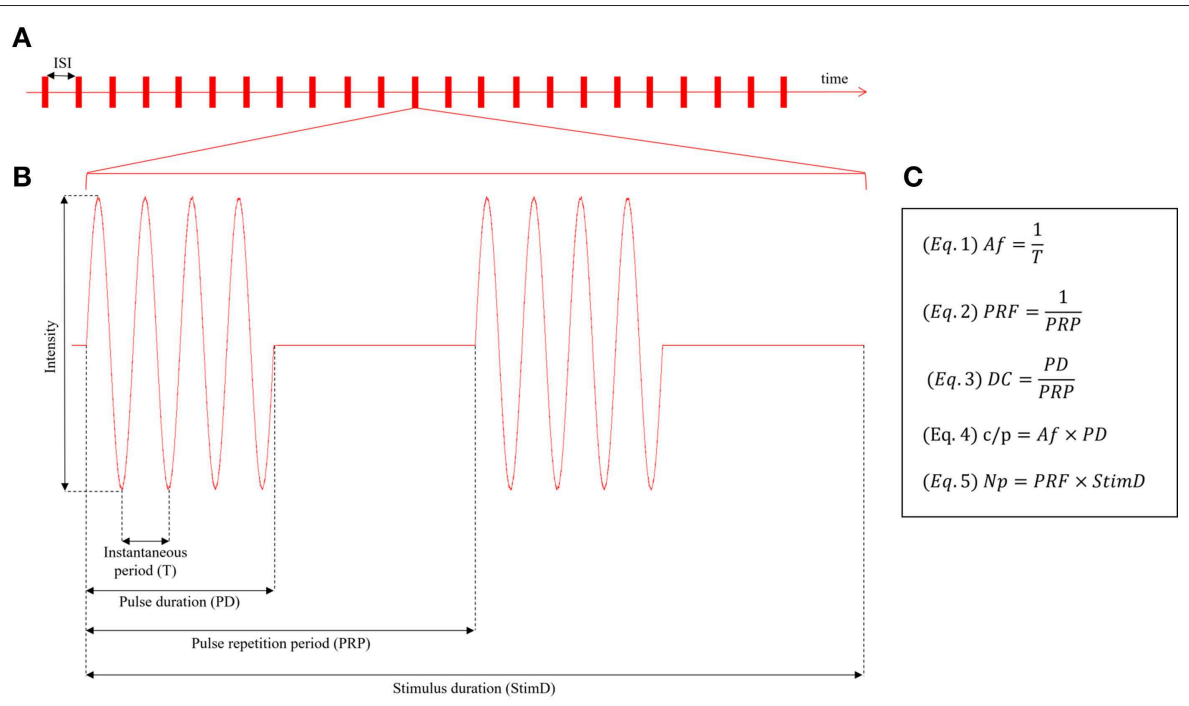

FIGURE 3 | (A) Intermittent protocol stimulation. The single sonications are followed by pauses, defined inter stimulation interval (ISI). (B) Pulsed paradigm of stimulation, defined by the following parameters: Intensity of stimulation, instantaneous period (T), pulse duration (PD), pulse repetition period (PRP), stimulus duration (StimD). (C) Fundamental equations for the stimulation protocol description: Equation (1) = Acoustic frequency (Af), Equation (2) = pulse repetition frequency (PRF), Equation (3) = duty cycle (DC), Equation (4) = cycles per pulse (c/p), Equation (5) = number of pulses (Np).

\section{Focused Ultrasound for Targeted Drug Delivery}

Focused ultrasound technique can be used also to facilitate drugs delivery in a specific brain area. Until now the most explored application is chemotherapy delivering. However, this versatile technique could be applied for neuromodulation purposes, with different mechanisms.

The first mechanism is a focal blood-brain barrier (BBB) opening, through a transient opening of endothelial tight junctions. Indeed, both animal (44, 45) and human (46) studies showed that FUS in combination with microbubbles administered intravenously can open the BBB, in a targeted, noninvasive, safe, and reversible manner. This technique could be used for targeted neuromodulation, with therapy which doesn't cross the BBB. For example Wang et al. (47) showed that it is possible to facilitate gene therapy delivery with recombinant adeno-associated virus, in a non-invasive way, through focused ultrasound targeted $\mathrm{BBB}$ opening, with potential applications for optogenetics (48) neuromodulation.

The second system is the local release of drugs, minimizing the effect on other brain areas. Indeed, focused ultrasound can be used to locally release drugs which are administered into the bloodstream through a vehicle (e.g., microbubble, liposome) sensitive to local temperature or pressure changes (49).

\section{METHODS}

The literature search methods included the PubMed/MEDLINE databases with the following research string, in Nov 2018: ("Neuromodulation" OR "Brain Stimulation") AND ("focused ultrasound" OR HIFU OR LIFU OR Low-intensity focused ultrasound). After abstract reading and screening, only human studies which described focused ultrasound neuromodulation approaches were included in the present review. In addition to the search protocol described, further articles suggested by experts in the field where read and screened (Table 1).

\section{RESULTS}

\section{Physiological Effects in Normal Subjects}

Legon et al. (10) used tFUS to target the human primary somatosensory cortex (S1), showing that tFUS significantly decreased the amplitudes of somatosensory evoked potentials elicited by median nerve stimulation. Furthermore, tFUS significantly modulated the spectral content of sensory-evoked brain oscillations and enhanced the performance on sensory discrimination tasks. The neurophysiologic effects had a spatial resolution of about $1 \mathrm{~cm}$ or less.

In another study, tFUS altered EEG intrinsic oscillatory dynamics, preferentially affecting the phase distribution of beta band and modulated the phase rate across beta and gamma frequencies. Furthermore, tFUS affected the phase distributions in the beta band of the early but not of the late components of somatosensory evoked potentials, suggesting a spatial specificity. This hypothesis was supported by the loss of neuromodulatory effects after the displacement of the transducer $1 \mathrm{~cm}$ laterally from the original cortical target (39).

Primary (SI) and secondary (SII) somatosensory cortical areas of the hand were targeted in a study by Lee et al. (50), in which two transducers were used. The areas were stimulated separately or simultaneously, under neuronavigation guide. tFUS elicited various types of tactile sensations in the contralateral hand/arm regions. The effects were transient and reversible, and the stimulation resulted safe, as assessed by repeated clinical and neuroradiological evaluations. In addition this study showed, the 
TABLE 1 | tFUS and tUS neuromodulation studies.

\begin{tabular}{|c|c|c|c|c|c|c|c|c|c|c|}
\hline References & Device & $\begin{array}{l}\text { N. of } \\
\text { subjects }\end{array}$ & $\begin{array}{l}\text { Disease } \\
\text { type/healthy } \\
\text { subjects }\end{array}$ & $\begin{array}{l}\text { Study } \\
\text { design }\end{array}$ & $\begin{array}{l}\text { Stimulation } \\
\text { target }\end{array}$ & $\begin{array}{l}\text { Protocol } \\
\text { duration }\end{array}$ & $\begin{array}{l}\text { Ultrasound } \\
\text { parameters }\end{array}$ & Energy & Results & Adverse events \\
\hline Ai et al. (33) & $\begin{array}{l}\text { Custom-made, } \\
\text { single-element FUS } \\
\text { transducer; } \\
\text { Af: } 0.50 \mathrm{MHz} \text { Diameter } \\
30 \mathrm{~mm} \text {, focal length } 30 \mathrm{~mm} \text {, } \\
7 \mathrm{~T} \text { MRI compatible } \\
\text { Focused, Pulsed }\end{array}$ & 5 & $\begin{array}{l}\text { Healthy } \\
\text { volunteers }\end{array}$ & $\begin{array}{l}\text { Within- } \\
\text { subjects, } \\
\text { sham- } \\
\text { controlled } \\
\text { study }\end{array}$ & $\begin{array}{l}\text { Primary } \\
\text { motor cortex } \\
\text { (tFUS paired with } \\
\text { high field } 7 \mathrm{~T} \text { fMRI } \\
\text { targeted on the } \\
\text { dominant thumb } \\
\text { BOLD representation }\end{array}$ & $\begin{array}{l}54 \text { stimuli, ISI } 5.5 \mathrm{~s} \\
\text { n) }\end{array}$ & $\begin{array}{l}\text { Af: } 0.50 \mathrm{MHz} \\
\text { PD: } 0.36 \mathrm{~ms} ; \\
\text { PRF: } 1 \mathrm{kHz} ; \\
\text { Np: } 500 ; \text { DC: } \\
\text { 36\%; } \\
\text { c/p: } 180 ; \text { StimD: } \\
500 \mathrm{~ms}\end{array}$ & $\begin{array}{l}\text { ISPPA: } 16.95 \\
\text { W/cm²; } \\
\text { MI: } 0.97\end{array}$ & $\begin{array}{l}\text { tFUS increased } \\
\text { BOLD activation } \\
\text { volumes } \\
\text { generated during a } \\
\text { cued tapping task. } \\
\text { The effect was } \\
\text { spatially confined } \\
\text { to the sonicated } \\
\text { area. No } \\
\text { detectable effects } \\
\text { on SMA and PMd. }\end{array}$ & $\begin{array}{l}\text { No auditory or } \\
\text { tactile sensation }\end{array}$ \\
\hline $\begin{array}{l}\text { Legon et al. } \\
\text { (34) }\end{array}$ & $\begin{array}{l}\text { Custom- designed, } \\
\text { single-element FUS } \\
\text { transducer; } \\
\text { Af: }^{0} 0.50 \mathrm{MHz} \\
\text { Height } 1.25 \mathrm{~cm} \text {, aperture } \\
30 \mathrm{~mm} \text {, focal length } 22 \mathrm{~mm} \text {, } \\
\text { Attached at the center of a } \\
\text { TMS } 8 \text {-coil (Magstim Inc., } \\
\text { UK) for concurrent and } \\
\text { concentric } \\
\text { tFUS/TMS delivery } \\
\text { Focused, Pulsed }\end{array}$ & $\begin{array}{l}12 \text { (exp. 1) } \\
10 \text { (exp. 2) } \\
28 \text { (exp. 3) }\end{array}$ & $\begin{array}{l}\text { Healthy } \\
\text { volunteers }\end{array}$ & $\begin{array}{l}\text { Within- } \\
\text { subjects, } \\
\text { sham- } \\
\text { controlled } \\
\text { study }\end{array}$ & $\begin{array}{l}\text { Primary } \\
\text { motor cortex } \\
\text { (Exp 1-2: } \\
\text { dominant FDI } \\
\text { hotspot; Exp 3: } \\
\text { dominant } \\
\text { APB hotspot) }\end{array}$ & $\begin{array}{l}\text { Exp1: } 10 \\
\text { tFUS/TMS stimuli } \\
\text { from RMT-20\% to } \\
100 \% \text { stimulator } \\
\text { output, in } \\
\text { increments of } 5 \% \text {, } \\
\text { ISI of } 10 \text { seconds) } \\
\text { Exp2: } 10 \\
\text { tFUS/TMS } \\
\text { stimulations every } \\
10 \text { s for each TMS } \\
\text { paired-pulse ISI } \\
\text { from } 1 \text { to } 15 \text { ms. } \\
\text { Exp3: } 100 \text { stimuli } \\
\text { at random time } \\
\text { intervals between } \\
3 \text { and } 6 \mathrm{~s}\end{array}$ & $\begin{array}{l}\text { Af: } 0.50 \mathrm{MHz} \\
\text { PD: } 0.36 \mathrm{~ms} ; \\
\text { PRF: } 1 \mathrm{kHz} ; \\
\text { Np: } 500 ; \mathrm{DC} \text { : } \\
36 \% \text {; } \\
\text { c/p: } 180 ; \\
\text { StimD: } 500 \mathrm{~ms} \\
\text { tFUS } 100 \mathrm{~ms} \text { prior } \\
\text { to: the TMS pulse } \\
\text { (exp. 1), to the CS } \\
\text { (exp. 2) and to the } \\
\text { visual stimulus } \\
\text { (exp. 3) }\end{array}$ & $\begin{array}{l}\text { ISPPA: } 17.12 \\
\text { W/cm²; } \\
\text { ISPTA: } 6.16 \\
\text { W/cm²; } \\
\text { MI: } 0.9\end{array}$ & $\begin{array}{l}\text { Concentric and } \\
\text { concurrent } \\
\text { tFUS/TMS on M1 } \\
\text { inhibited the } \\
\text { amplitude of } \\
\text { single-pulse } \\
\text { MEPs, attenuated } \\
\text { intracortical } \\
\text { facilitation, did not } \\
\text { affect intracortical } \\
\text { inhibition and } \\
\text { significantly } \\
\text { reduced reaction } \\
\text { time in a motor } \\
\text { task. }\end{array}$ & $\begin{array}{l}\text { Mild and moderate } \\
\text { symptoms such as } \\
\text { neck pain, } \\
\text { sleepiness, muscle } \\
\text { twitches, itchiness } \\
\text { and headache } \\
\text { (assessed by } \\
\text { questionnaire). No } \\
\text { severe symptoms } \\
\text { reported. }\end{array}$ \\
\hline $\begin{array}{l}\text { Legon et al. } \\
\text { (35) }\end{array}$ & $\begin{array}{l}\text { Custom-designed, } \\
\text { single-element FUS } \\
\text { transducer (Ultran Group, } \\
\text { Inc., State College, PA); } \\
\text { Af: } 0.50 \mathrm{MHz} \\
\text { Aperture } 63 \mathrm{~mm} \text {, focal } \\
\text { length } 70.92 \mathrm{~mm}(55 \mathrm{~mm} \\
\text { from exit plane), f\# } 1.13 \\
\text { Focused, Pulsed }\end{array}$ & $\begin{array}{l}20 \text { (exp. 1) } \\
20 \text { (exp. 2) }\end{array}$ & $\begin{array}{l}\text { Healthy } \\
\text { volunteers }\end{array}$ & $\begin{array}{l}\text { Within- } \\
\text { subjects, } \\
\text { sham- } \\
\text { controlled } \\
\text { study }\end{array}$ & $\begin{array}{l}\text { Unilateral } \\
\text { sensory thalamus } \\
\text { targeted through a } \\
\text { neuronavigation } \\
\text { system from the } \\
\text { individual MRI }\end{array}$ & $\begin{array}{l}\text { Exp1: } 300 \text { stimuli, } \\
\text { ISI } 4 \text { s } \\
\text { Exp2: } 90 \text { stimuli }\end{array}$ & $\begin{array}{l}\text { Af: } 0.50 \mathrm{MHz} \\
\text { PD: } 0.36 \mathrm{~ms} ; \\
\text { PRF: } 1 \mathrm{kHz} ; \\
\text { Np: } 500 ; \mathrm{DC} \text { : } \\
\text { 36\%; } \\
\text { c/p: } 180 ; \\
\text { StimD: } 500 \mathrm{~ms} \\
\text { Median nerve } \\
\text { stimuli time-locked } \\
\text { to occur } 100 \mathrm{~ms} \\
\text { after the onset of } \\
\text { tFUS waveforms }\end{array}$ & $\begin{array}{l}\text { ISPPA: } 14.56 \\
\text { W/cm²; } \\
\text { MI: } 0.89 \\
\text { After bone } \\
\text { transmission: } \\
\text { ISPPA: } 7.03 ; \\
\text { W/cm²; } \\
\text { Ml: } 0.56\end{array}$ & $\begin{array}{l}\text { tFUS decreased } \\
\text { P14 SEP } \\
\text { amplitude. } \\
\text { Decrease in ability } \\
\text { in a tactile } \\
\text { judgement task. } \\
\text { Effect upon } \\
\text { cortical oscillatory } \\
\text { dynamics }\end{array}$ & Not available \\
\hline
\end{tabular}


TABLE 1 | Continued

\begin{tabular}{|c|c|c|c|c|c|c|c|c|c|c|}
\hline References & Device & $\begin{array}{l}\text { N. of } \\
\text { subjects }\end{array}$ & $\begin{array}{l}\text { Disease } \\
\text { type/healthy } \\
\text { subjects }\end{array}$ & $\begin{array}{l}\text { Study } \\
\text { design }\end{array}$ & $\begin{array}{l}\text { Stimulation } \\
\text { target }\end{array}$ & $\begin{array}{l}\text { Protocol } \\
\text { duration }\end{array}$ & $\begin{array}{l}\text { Ultrasound } \\
\text { parameters }\end{array}$ & Energy & Results & Adverse events \\
\hline $\begin{array}{l}\text { Leo et al. } \\
\text { (36) }\end{array}$ & 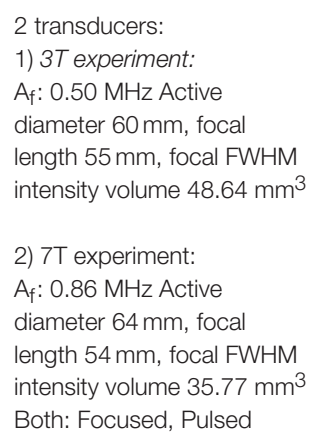 & $\begin{array}{l}6 \text { (3T exp.) } \\
1 \text { (7T exp.) }\end{array}$ & $\begin{array}{l}\text { Healthy } \\
\text { volunteers }\end{array}$ & $\begin{array}{l}\text { Pre-post } \\
\text { interventional } \\
\text { study }\end{array}$ & $\begin{array}{l}\text { 3T experiment: } \\
\text { Primary motor } \\
\text { cortex hand knob } \\
\text { of the dominant } \\
\text { hemisphere } 7 \mathrm{~T} \\
\text { experiment: Left } \\
\text { head of } \\
\text { the caudate }\end{array}$ & $\begin{array}{l}\text { 3T experiment: } \\
90 \text { stimuli, ISI } \\
12-14 \mathrm{~s} \\
7 \mathrm{~T} \text { experiment: } \\
5 \text { off/on cycles, } \\
\text { stimulation } \\
\text { delivered at ISI } \cong \\
12 \mathrm{~s} \text { during on } \\
\text { cycles }\end{array}$ & $\begin{array}{l}\text { 3T experiment: } \mathrm{A}_{f} \text { : } \\
\text { 0.50 MHz; } \\
\text { PRF: } 1 \mathrm{kHz} ; \\
\text { Np: } 500 ; \mathrm{DC} \text { : } \\
\text { 36\%; } \\
\text { c/p: } 180 \text {; } \\
\text { StimD: } 500 \mathrm{~ms} 7 \mathrm{~T} \\
\text { experiment: } \\
\text { Af: } 0.86 \mathrm{MHz} \text {; } \\
\text { PRF: } 1 \mathrm{kHz} ; \\
\text { DC: } 50 \% \text {; } / \mathrm{p} \text { : } \\
\text { 420; } \\
\text { StimD: } 500 \mathrm{~ms}\end{array}$ & $\begin{array}{l}\text { ISPPA: } 6 \mathrm{~W} / \mathrm{cm}^{2} \\
\text { (after bone } \\
\text { transmission) }\end{array}$ & $\begin{array}{l}\text { tFUS induced } \\
\text { BOLD fMRI signals } \\
\text { in the targeted } \\
\text { cortical regions (in } \\
3 \text { of } 6 \text { subjects) } \\
\text { and in the targeted } \\
\text { subcortical region }\end{array}$ & Not available \\
\hline $\begin{array}{l}\text { Lee et al. } \\
\text { (37) }\end{array}$ & $\begin{array}{l}\text { MRI-compatible } \\
\text { FUS transducer } \\
\text { Af: } 0.27 \mathrm{MHz} \\
\text { Focal length } 3 \mathrm{~cm} \text {, acoustic } \\
\text { focus } 3 \mathrm{~mm} \text { (diameter) and } \\
17 \mathrm{~mm} \text { (length) Focused, } \\
\text { Pulsed }\end{array}$ & $\begin{array}{l}19 \text { (exp. 1) } \\
10 \text { (exp. 2) }\end{array}$ & $\begin{array}{l}\text { Healthy } \\
\text { volunteers }\end{array}$ & $\begin{array}{l}\text { Within- } \\
\text { subjects, } \\
\text { single- } \\
\text { blind, } \\
\text { sham- } \\
\text { controlled } \\
\text { study }\end{array}$ & $\begin{array}{l}\text { Primary visual } \\
\text { cortex, under } 3 T \\
\text { MRI guidance }\end{array}$ & $\begin{array}{l}\text { Exp.1: } \\
50 \text { stimuli, ISI } 13 \mathrm{~s} \\
\text { Exp.2: } \\
50 \text { stimuli, ISI } 2.5 \mathrm{~s}\end{array}$ & $\begin{array}{l}\text { Af: } 0.27 \mathrm{MHz} \text {; } \\
\text { PRF: } 500 \mathrm{~Hz} \text {; PD: } \\
1 \mathrm{~ms} ; \\
\text { DC: } 50 \% \text {; StimD: } \\
300 \mathrm{~ms}\end{array}$ & $\begin{array}{l}\text { ISPPA: } 16.6 \\
\text { W/cm2 } \\
\text { Estimates at the } \\
\text { target location: } \\
\text { ISPPA: mean } 3 \\
\text { W/cm2; } \\
\text { Ml: mean } 0.6\end{array}$ & $\begin{array}{l}\text { tFUS induced } \\
\text { BOLD fMRI signals } \\
\text { in V1 and other } \\
\text { visual areas, } \\
\text { elicited } \\
\text { phosphenes and } \\
\text { elicited cortical } \\
\text { evoked EEG } \\
\text { potentials similar } \\
\text { to the classical } \\
\text { VEP generated by } \\
\text { photic stimulation }\end{array}$ & $\begin{array}{l}\text { No adverse } \\
\text { effects, as } \\
\text { assessed by } \\
\text { neurological } \\
\text { examination, } \\
\text { anatomical MRI (at } \\
3 \text { time points) and } \\
\text { follow-up } \\
\text { telephone } \\
\text { interviews (after } 2 \\
\text { months) }\end{array}$ \\
\hline $\begin{array}{l}\text { Lee et al. } \\
\text { (37) }\end{array}$ & $\begin{array}{l}\text { Two sets of single-element } \\
\text { FUS transducers (Ultran } \\
\text { Group Ltd, State } \\
\text { College, PA) } \\
\text { Af: } 0.21 \mathrm{MHz} \text { Shape: } \\
\text { segmented-spheres Outer } \\
\text { diameter (OD):30 mm Focal } \\
\text { distance: } 25 \mathrm{~mm} \text {. Each } \\
\text { transducer was affixed to an } \\
\text { applicator (Zamerican, } \\
\text { Zacuto, Chicago, IL) } \\
\text { mounted on a helmet } \\
\text { (modified from Giro Section } \\
\text { Helmet, Santa Cruz, CA) } \\
\text { Focused, Pulsed }\end{array}$ & 10 & $\begin{array}{l}\text { Healthy } \\
\text { volunteers }\end{array}$ & $\begin{array}{l}\text { Within- } \\
\text { subjects, } \\
\text { double } \\
\text { blind,sham- } \\
\text { controlled } \\
\text { study }\end{array}$ & $\begin{array}{l}\text { Left primary and } \\
\text { secondary } \\
\text { somatosensory } \\
\text { cortex (areas of } \\
\text { the hand, } \\
\text { separately or } \\
\text { simultaneously } \\
\text { stimulated under } \\
\text { multi-modal } \\
\text { neuroimage- } \\
\text { guidance) }\end{array}$ & $\begin{array}{l}20 \text { stimuli for each } \\
\text { session (4 } \\
\text { sessions) }\end{array}$ & $\begin{array}{l}\text { Af: } 0.21 \mathrm{MHz} ; \\
\text { PRF: } 500 \mathrm{~Hz} ; \\
\text { PD: } 1 \mathrm{~ms} ; \\
\text { DC: } 50 \% \text {; StimD: } \\
500 \mathrm{~ms}\end{array}$ & $\begin{array}{l}\text { ISPPA: } 35.0 \\
\text { W/cm²; } \\
\text { ISPTA: } 17.5 \\
\text { W/cm } 2 \\
\text { Estimates at the } \\
\text { target location: } \\
\text { ISPPA: } 7.0-8.8 \\
\text { W/cm } 2 \\
\text { ISPTA: } 3.5-4.4 \\
\text { W/cm } 2\end{array}$ & $\begin{array}{l}\text { tFUS of either } \\
\text { primary and } \\
\text { secondary } \\
\text { somatosensory } \\
\text { cortex, stimulated } \\
\text { separately or } \\
\text { simultaneously, } \\
\text { eliciited tactile } \\
\text { sensations from } \\
\text { the contralateral } \\
\text { hand/arm areas }\end{array}$ & $\begin{array}{l}\text { No abnormal } \\
\text { findings post-tFUS } \\
\text { (assessed by } \\
\text { neurological } \\
\text { examination, } \\
\text { MMSE, anatomical } \\
\text { MRI on the same } \\
\text { day, at } 2 \text { weeks } \\
\text { and } 4 \text { weeks, and } \\
\text { by telephone } \\
\text { interview at } 2 \\
\text { months after the } \\
\text { sonications) }\end{array}$ \\
\hline
\end{tabular}


TABLE 1 | Continued

\begin{tabular}{|c|c|c|c|c|c|c|c|c|c|c|}
\hline References & Device & $\begin{array}{l}\text { N. of } \\
\text { subjects }\end{array}$ & $\begin{array}{l}\text { Disease } \\
\text { type/healthy } \\
\text { subjects }\end{array}$ & $\begin{array}{l}\text { Study } \\
\text { design }\end{array}$ & $\begin{array}{l}\text { Stimulation } \\
\text { target }\end{array}$ & $\begin{array}{l}\text { Protocol } \\
\text { duration }\end{array}$ & $\begin{array}{l}\text { Ultrasound } \\
\text { parameters }\end{array}$ & Energy & Results & Adverse events \\
\hline $\begin{array}{l}\text { Monti et al. } \\
\text { (11) }\end{array}$ & $\begin{array}{l}\text { BXPulsar 1001, } \\
\text { Brainsonix Inc. } \\
\text { Single-element spherical } \\
\text { transducer; } \\
\mathrm{A}_{\mathrm{f}} \text { : } 0.65 \mathrm{MHz} \text { Diameter and } \\
\text { radius of curvature } 71.5 \mathrm{~mm} \\
\text { Focused, Pulsed }\end{array}$ & 1 & $\begin{array}{l}\text { Post- } \\
\text { traumatic } \\
\text { disorder of } \\
\text { consciousness } \\
\text { (minimally } \\
\text { conscious } \\
\text { state) } 19 \text { days } \\
\text { post-injury }\end{array}$ & $\begin{array}{l}\text { Case } \\
\text { report, part } \\
\text { of an } \\
\text { ongoing } \\
\text { clinical trial } \\
\text { (12) }\end{array}$ & $\begin{array}{l}\text { Thalamus } \\
\text { (MRI-guided by a } \\
3 \text { Tesla Magnetom } \\
\text { Tim Trio } \\
\text { MR scanner) }\end{array}$ & $\begin{array}{l}10 \text { sonications, } \\
30 \text { s each, } \\
\text { separated by } 30 \text { s } \\
\text { pause intervals }\end{array}$ & $\begin{array}{l}A_{f}: 0.65 \mathrm{MHz} \\
\text { PD: } 0.5 \mathrm{~ms} ; \\
\text { PRF: } 100 \mathrm{~Hz}\end{array}$ & $\begin{array}{l}\text { ISPTA: } 720 \\
\mathrm{~mW} / \mathrm{cm}^{2}\end{array}$ & $\begin{array}{l}\text { Emergence from } \\
\text { minimally } \\
\text { conscious state }\end{array}$ & $\begin{array}{l}\text { Clinical } \\
\text { improvement } \\
\text { suggested that the } \\
\text { procedure was } \\
\text { safe and } \\
\text { well-tolerated }\end{array}$ \\
\hline $\begin{array}{l}\text { Lee et al. } \\
\text { (38) }\end{array}$ & 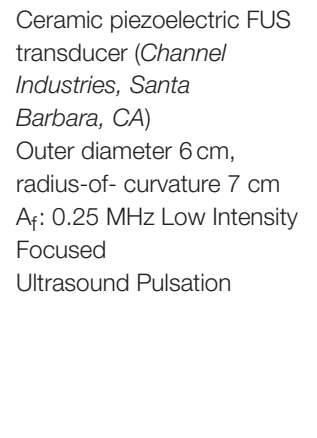 & $\begin{array}{l}12(\exp .1) \\
6(\exp .2)\end{array}$ & $\begin{array}{l}\text { Healthy } \\
\text { volunteers }\end{array}$ & $\begin{array}{l}\text { Within- } \\
\text { subjects, } \\
\text { sham- } \\
\text { controlled } \\
\text { study }\end{array}$ & $\begin{array}{l}\text { Primary } \\
\text { somatosensory } \\
\text { cortex (hand area) } \\
\text { under subject- } \\
\text { specific image- } \\
\text { guidance }\end{array}$ & $\begin{array}{l}\text { (Exp. 1): } \\
200 \text { stimuli, ISI } 3 \text { s } \\
\text { (Exp. 2): } \\
100 \text { stimuli, ISI } \\
\cong 2 \mathrm{~s}\end{array}$ & $\begin{array}{l}\text { Af: } 0.25 \mathrm{MHz} ; \\
\text { PRF: } 500 \mathrm{~Hz} ; \\
\text { Tone-burst- } \\
\text { duration: } 1 \mathrm{~ms} ; \\
\text { DC: } 50 \% \text {; } \\
\text { StimD: } 300 \mathrm{~ms}\end{array}$ & $\begin{array}{l}\text { ISPPA: } 3 \mathrm{~W} / \mathrm{cm}^{2} \\
\text { Estimated ISPPA at } \\
\text { the target: } \\
0.7 \pm 0.5 \mathrm{~W} / \mathrm{cm}^{2}\end{array}$ & $\begin{array}{l}\text { tFUS elicited } \\
\text { transient tactile } \\
\text { sensations on the } \\
\text { hand and arm area } \\
\text { contralateral to the } \\
\text { sonicated } \\
\text { hemisphere, with } \\
\text { anatomical } \\
\text { specificity of up to } \\
\text { a finger. EEG } \\
\text { showed } \\
\text { sonication-specific } \\
\text { evoked potentials. }\end{array}$ & $\begin{array}{l}\text { No adverse } \\
\text { effects, as } \\
\text { assessed by } \\
\text { neurological } \\
\text { examination, } \\
\text { anatomical MRI (at } \\
3 \text { time points) and } \\
\text { follow-up } \\
\text { telephone } \\
\text { interviews (after } 2 \\
\text { months) }\end{array}$ \\
\hline $\begin{array}{l}\text { Mueller } \\
\text { et al. (39) }\end{array}$ & $\begin{array}{l}\text { Two-channel, } 2 \mathrm{MHz} \\
\text { function generator (BK } \\
\text { Precision Instruments) } \\
\text { delivered at } 0.5 \mathrm{MHz} \\
\text { Focused, pulsed }\end{array}$ & $\begin{array}{l}18 \text { (exp. 1) } \\
7 \\
\text { (exp. 2) }\end{array}$ & $\begin{array}{l}\text { Healthy } \\
\text { volunteers }\end{array}$ & $\begin{array}{l}\text { Within- } \\
\text { subjects, } \\
\text { sham- } \\
\text { controlled } \\
\text { study }\end{array}$ & $\begin{array}{l}\text { Exp.1 } \\
\text { Somatosensory } \\
\text { cortex (CP3) } \\
\text { Exp. } 21 \mathrm{~cm} \text { laterally }\end{array}$ & 120 stimuli, ISI 6 s & $\begin{array}{l}\text { Af: } 0.50 \mathrm{MHz} ; \\
\text { PD: } 0.36 \mathrm{~ms} ; \\
\text { PRF: } 1 \mathrm{kHz} ; \\
\text { Np: } 500 ; \mathrm{c} / \mathrm{p}: 180 ; \\
\text { StimD: } 500 \mathrm{~ms}\end{array}$ & $\begin{array}{l}\text { ISPPA: } 23.87 \\
\text { W/cm²; } \\
\text { MI: } 1.13\end{array}$ & $\begin{array}{l}\text { tFUS altered EEG } \\
\text { beta phase and } \\
\text { modulated the } \\
\text { phase rate across } \\
\text { beta and } \\
\text { gamma frequencies. } \\
\text { tFUS affected } \\
\text { phase distributions } \\
\text { in the beta band of } \\
\text { early SEP } \\
\text { components. } \\
\text { Neuromodulatory } \\
\text { effects were lost } \\
\text { when the } \\
\text { transducer was } \\
\text { displaced } 1 \mathrm{~cm} \\
\text { laterally from the } \\
\text { original } \\
\text { cortical target. }\end{array}$ & Not available \\
\hline
\end{tabular}


TABLE 1 | Continued

\begin{tabular}{|c|c|c|c|c|c|c|c|c|c|c|}
\hline References & Device & $\begin{array}{l}\text { N. of } \\
\text { subjects }\end{array}$ & $\begin{array}{l}\text { Disease } \\
\text { type/healthy } \\
\text { subjects }\end{array}$ & $\begin{array}{l}\text { Study } \\
\text { design }\end{array}$ & $\begin{array}{l}\text { Stimulation } \\
\text { target }\end{array}$ & $\begin{array}{l}\text { Protocol } \\
\text { duration }\end{array}$ & $\begin{array}{l}\text { Ultrasound } \\
\text { parameters }\end{array}$ & Energy & Results & Adverse events \\
\hline $\begin{array}{l}\text { Legon et al. } \\
\text { (10) }\end{array}$ & $\begin{array}{l}\text { Custom-made, } \\
\text { single-element FUS } \\
\text { transducer; } \\
\text { Af: }_{f} \text { 0.50 MHz Diameter } \\
30 \mathrm{~mm} \text {, focal length } 30 \mathrm{~mm} \\
\text { Focused, Pulsed }\end{array}$ & $\begin{array}{l}10 \text { (exp. 1) } \\
8 \text { (exp. 2) } \\
12 \text { (exp. 3) } \\
12 \text { (exp. 4) }\end{array}$ & $\begin{array}{l}\text { Healthy } \\
\text { volunteers }\end{array}$ & $\begin{array}{l}\text { Within- } \\
\text { subjects, } \\
\text { sham- } \\
\text { controlled } \\
\text { study }\end{array}$ & $\begin{array}{l}\text { Primary } \\
\text { somatosensory } \\
\text { cortex (crown of } \\
\text { the postcentral } \\
\text { gyrus and } \\
\text { posterior wall of } \\
\text { the central sulcus, } \\
\text { encephalographic } \\
\text { electrode CP3) }\end{array}$ & $\begin{array}{l}\text { Exp } 1 \text { and 2: } 120 \\
\text { stimuli, ISI } 6 \mathrm{~s} \\
\text { Exp 3: } 90 \text { stimuli } \\
100 \mathrm{~ms} \text { before } \\
\text { each task } \\
\text { Exp4: 120, ISI 6s }\end{array}$ & $\begin{array}{l}\text { Af: } 0.50 \mathrm{MHz} ; \\
\text { PD: } 0.36 \mathrm{~ms} ; \\
\text { PRF: } 1 \mathrm{kHz} ; \\
\text { Np: } 500 ; \mathrm{DC} \text { : } \\
\text { 36\%; } \\
\text { c/p: } 180 ; \\
\text { StimD: } 500 \mathrm{~ms} \\
\text { Median nerve } \\
\text { stimuli time-locked } \\
\text { to occur } 100 \mathrm{~ms} \\
\text { after the onset of } \\
\text { tFUS waveforms }\end{array}$ & 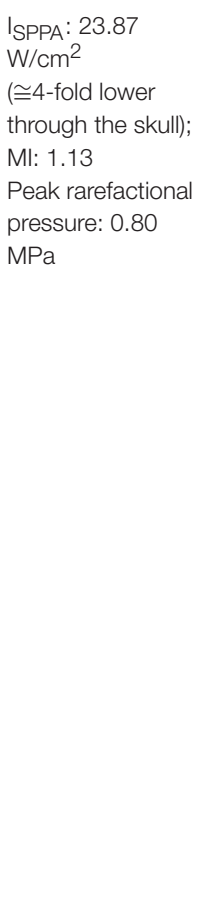 & $\begin{array}{l}\text { Exp1. A: tFUS } \\
\text { significantly } \\
\text { attenuated the } \\
\text { amplitudes of } \\
\text { somatosensory } \\
\text { evoked potentials } \\
\text { B: tFUS } \\
\text { significantly } \\
\text { modulated the } \\
\text { spectral content of } \\
\text { sensory-evoked } \\
\text { brain oscillations } \\
\text { Exp2. tFUS } \\
\text { modulation of } \\
\text { brain activity is } \\
\text { spatially restricted } \\
\text { (@1 cm or less) } \\
\text { Exp3 and 4. tFUS } \\
\text { significantly } \\
\text { enhanced } \\
\text { performance on } \\
\text { sensory } \\
\text { discrimination } \\
\text { tasks without } \\
\text { affecting task } \\
\text { attention or } \\
\text { response bias. }\end{array}$ & $\begin{array}{l}\text { No thermal or } \\
\text { mechanical } \\
\text { sensation }\end{array}$ \\
\hline $\begin{array}{l}\text { Gibson } \\
\text { et al. (40) }\end{array}$ & $\begin{array}{l}\text { tUS: Phillips CX50 } \\
\text { Diagnostic System, with a } \\
\text { Phillips S5-1 broadband } \\
\text { plane sector transducer } \\
\text { array; aperture } 20.3 \mathrm{~cm} \text {, } \\
\text { frequency range } 1-5 \mathrm{MHz} \text {. } \\
\text { TMS: } \\
\text { neuronavigation-assisted } \\
\text { eXemia TMS system } \\
\text { (Nextstim Ltd., Helsinki, } \\
\text { Finland) with a } 70 \\
\text { mm 8-coil. } \\
\text { Unfocused, Continuous }\end{array}$ & $\begin{array}{l}21 \text { (active } \\
\text { stim) } \\
22 \text { (sham } \\
\text { stim) }\end{array}$ & $\begin{array}{l}\text { Healthy } \\
\text { volunteers }\end{array}$ & $\begin{array}{l}\text { Between- } \\
\text { subjects, } \\
\text { single- } \\
\text { blind, } \\
\text { sham- } \\
\text { controlled } \\
\text { study }\end{array}$ & $\begin{array}{l}\text { Primary } \\
\text { motor cortex } \\
\text { (abductor pollicis } \\
\text { brevis } \\
\text { motor hotspot) }\end{array}$ & $2 \mathrm{~min}$ & $\begin{array}{l}\text { Af: } 2.32 \mathrm{MHz} ; \\
\text { HGen, B-mode; } \\
\text { Harmonics: on; } \\
\text { DC: }<1 \% \text {; Focal } \\
\text { depth: } 10 \mathrm{~cm}\end{array}$ & $\begin{array}{l}\text { Isppa: } 34.96 \\
\text { W/cm²; Ispta: } \\
132.85 \mathrm{~mW} / \mathrm{cm}^{2} ; \\
\text { Ml: } 0.67 \\
\text { Peak negative } \\
\text { pressure: } 1.02 \\
\text { MPa (in degassed } \\
\text { water) }\end{array}$ & $\begin{array}{l}\text { tUS increased } \\
\text { cortical excitability } \\
\text { (average increase } \\
\text { in MEPs amplitude } \\
\text { of } 33.7 \% \text { at } 1 \text { min } \\
\text { and of } 32.2 \% \text { at } \\
6 \text { min post } \\
\text { stimulation. } \\
\text { No significant } \\
\text { differences at } 11 \\
\text { and } 16 \text { min } \\
\text { post stimulation). } \\
\text { No differences in } \\
\text { mood (assessed } \\
\text { by a brief } \\
\text { questionnaire on } \\
\text { subject well-being) }\end{array}$ & $\begin{array}{l}\text { No significant } \\
\text { differences in } \\
\text { sensations linked } \\
\text { tingling, itching } \\
\text { etc. (assessed by } \\
\text { questionnaires) } \\
\text { between active } \\
\text { and sham group }\end{array}$ \\
\hline
\end{tabular}




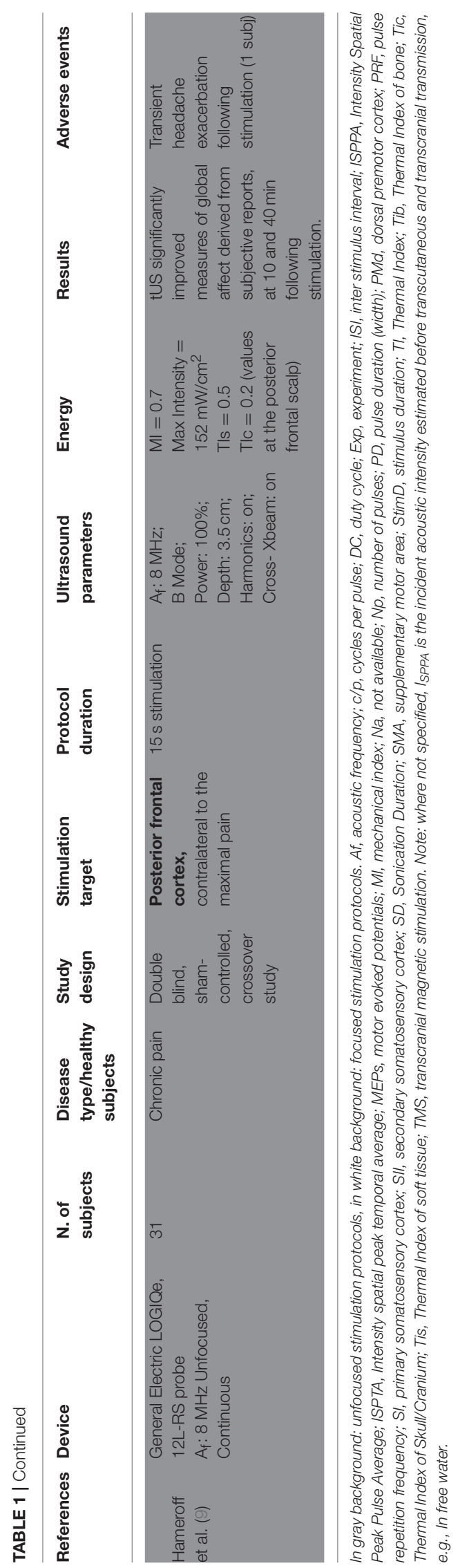

feasibility of the simultaneous stimulation of different human brain areas.

In Lee et al. (38), tFUS stimulation of the human somatosensory cortex elicited somatosensory sensations with anatomical specificity up to a finger, and evoked EEG potentials.

fMRI studies showed the effects of tFUS on cortical and subcortical brain areas, with the ability of high-resolution noninvasive functional brain mapping $(33,36,37)$.

Indeed, Leo et al. (36), demonstrated that tFUS stimulation of cortical (primary motor cortex) and subcortical (head of the caudate) areas can induce blood oxygen level dependent (BOLD) signals in $3 \mathrm{~T}$ and $7 \mathrm{~T}$ fMRI, respectively. More recently, pairing tFUS on human primary motor cortex (M1) with 7T BOLD fMRI signals in a cued finger tapping task study, Ai et al. (33) showed that tFUS selectively increases BOLD activation volumes of the target finger representation. These effects did not spatially overcome the sonicated area, and therefore did not involve other motor regions, such as supplementary motor area (SMA) and dorsal premotor cortex (PMd).

tFUS has been used also to target the human primary visual cortex (V1) Lee et al. (37) showed, on BOLD fMRI signals, that tFUS stimulation elicited the activation of a network of brain regions, including V1 and other areas involved in visual and higher-order cognitive processes. Furthermore, stimulation elicited perception of phosphenes and EEG evoked responses.

The effects of tFUS on corticospinal excitability have also been studied through transcranial magnetic stimulation (TMS). Combining a custom-made FUS transducer and a 8-shaped TMS coil, Legon et al. (34) assessed for the first time in humans the effect of concentric and concurrent tFUS/TMS stimulation on M1. The stimulation had an inhibitory effect on single-pulse MEPs and intracortical facilitation, and significantly decreased the reaction time in a motor task.

Legon et al. (35) tested the effects tFUS stimulation on sensory thalamus, that was targeted by a single-element focused ultrasound through a neuronavigation system based on the individual subject anatomical MRI. tFUS stimulation inhibited the P14 SEP, and was associated with a change in EEG oscillatory dynamics and to a reduced ability in a tactile judgement task. In addition, this study outlined the value of taking into account the individual skull morphology to produce safe and accurate stimulations.

In a recent single-blind, sham-controlled study (40), tUS was targeted to the motor cortex through a diagnostic imaging ultrasound system. The unfocused stimulation increased MEPs amplitude by $34 \%$ compared to baseline, and the increase was recorded up to $6 \mathrm{~min}$ after the stimulation. This short-term increase of motor cortex excitability contrasts with a previous findings of MEP inhibition during concurrent tFUS/TMS (34). As discussed by the authors, stimulation parameters and other methodological factors might explain the different findings.

\section{Therapeutic Application}

Despite several studies showed the neurological therapeutic applications of lesional FUS and FUS mediated BBB opening in different diseases like essential tremor (51-54), Parkinson's disease (55-57), depression $(58,59)$, obsessive-compulsive 
disorder $(60,61)$, neuropatic pain $(62,63)$, Alzheimer disease $(46,64)$, only two studies explored in humans the therapeutic efficacy of tUS (9) and tFUS (11) bioelectrical neuromodulation (Table 1).

Hameroff et al. (9) used a $8 \mathrm{MHz}$ unfocused transducer to study the effects of transcranial ultrasound stimulation (tUS) on mood, and global affect in 31 patients with chronic pain, in a double-blind, sham-controlled crossover study. Stimulation was targeted to the posterior frontal cortex, contralateral to the most severe pain. After the stimulation, a significant improvement in subjective parameters of global affect derived from the Visual Analog Mood Scale was found.

As part of an ongoing clinical trial on low intensity focused ultrasound in acute brain injury (12), Monti et al. (11) reported a case of emergence from minimally conscious state after low intensity non-invasive ultrasonic thalamic stimulation.

\section{Transcranial Focused vs. Unfocused Ultrasound Neuromodulation}

Despite transcranial focused ultrasound (tFUS) and transcranial unfocused ultrasound (tUS) neuromodulation techniques share the same basic mechanisms of action, when applied on the same target they can lead to quite different results.

These results are related to the intrinsic differences between the two techniques. The most important, one is the volume of the brain involved in the ultrasound field. It is intuitive that the volume of the brain involved in the focused or unfocused neuromodulation, and the underlying neural circuits, are crucial to determine the output of the tFUS or tUS neuromodulation. This has been supported also by experimental results, where tFUS and tUS were applied on the same target, the primary motor cortex: tUS increased MEPs amplitude (40) instead tFUS induced a MEP inhibition (34). In addition, the sonication delivered during the stimulus duration period, is generally continuous, without pauses, for tUS, and pulsed, characterized by pauses between the sonications, for tFUS. Low-intensity pulsed FUS is the most effective FUS technique for neuromodulation in both animal model $(5,6)$ and humans (Table 1). Instead, high intensity continuous FUS is widely used for therapeutic irreversible lesioning $(51,55,58,60)$.

\section{DISCUSSION}

Transcranial focused ultrasound is an emerging technique for non-invasive neurostimulation, with direct action on bioelettrical neural activity, and in addition could be used for targeted drug delivery.

\section{REFERENCES}

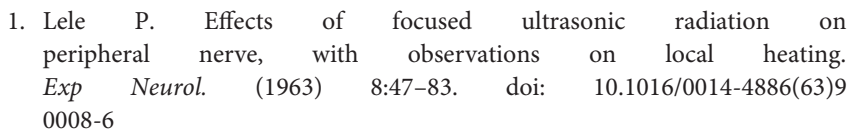

Compared to magnetic or electric non-invasive brain stimulation, this technique has a higher spatial resolution and can reach deep structures. In addition, animal studies suggest that, potentially, different sites of the central and peripheral nervous system can be targeted by this technique.

Even if still in a small number, the increasing interest in this technique, led to encouraging results in human studies. These preliminary human studies focused their attention on classic non-invasive neurostimulation targets, like the primary motor cortex, somatosensory area or primary visual cortex, with some studies that explored deep structures like thalamus $(11,35)$ or basal ganglia (36). All showed neurostimulation efficacy in terms of clinical, neurophysiological or functional neuroradiological outcomes (Table $\mathbf{1}$ ).

The data collected since now shows that this technique is safe and well-tolerated, when the stimulation parameters and protocol follow the available guidelines. In addition, tFUS can be also conducted without hair shaving (65). The majority of the studies reported no severe adverse effects. Mild and moderate symptoms are reported such as neck pain, sleepiness, muscle twitches, itchiness, and headache $(9,34)$ (Table 1). In future studies, proper assessments, aimed to define the safety parameters for tUS and tFUS, are needed. Finally, every tUS or tFUS protocol should explore the role of auditory confounding factors on the neural responses, in order to show that the effect of stimulation is the consequence only of the targeted area neuromodulation, and not due to an indirect auditory impact $(66,67)$.

Overall, the results up to now encourage the study of tUS and tFUS as non-invasive neuromodulatory techniques in humans. The high spatial resolution of tFUS and the possibility of stimulating cortical and deep brain regions suggest many potential applications, such as cortical and subcortical mapping, the study of functional connectivity, the modulation of neurotransmission. Regarding tUS as a potential neuromodulatory tool, noteworthy is the high accessibility of the devices, which are routinely used in health care settings. Further research is needed to clarify tUS and tFUS efficacy and underlying mechanisms, and to optimize stimulation parameters and targeting accuracy. The initial safety profiles seem promising. A rigorous approach must be maintained in order to ensure safe sonications.

\section{AUTHOR CONTRIBUTIONS}

LB: conception, organization, execution, and writing of the first draft. EF: execution, writing of the first draft, and review and critique. VD: conception, organization, and review and critique. 
4. Deffieux T, Younan Y, Wattiez N, Tanter M, Pouget P, Aubry JF. Low-intensity focused ultrasound modulates monkey visuomotor behavior. Curr Biol. (2013) 23:2430-3. doi: 10.1016/j.cub.2013. 10.029

5. Folloni D, Verhagen L, Mars RB, Fouragnan E, Constans C, Aubry J-F, et al. Manipulation of subcortical and deep cortical activity in the primate brain using transcranial focused ultrasound stimulation. Neuron. (2019) 101:110916.e1105. doi: 10.1016/j.neuron.2019.01.019

6. Dallapiazza RF, Timbie KF, Holmberg S, Gatesman J, Lopes MB, Price RJ, et al. Non-invasive neuromodulation and thalamic mapping with low-intensity focused ultrasound. J Neurosurg.(2018) 128:875-84. doi: 10.3171/2016.11.JNS16976

7. FRY WJ. Intense ultrasound in investigations of the central nervous system. Adv Biol Med Phys. (1958) 6:281-348.

8. Verhagen L, Gallea C, Folloni D, Constans C, Jensen DE, Ahnine H, et al. Offline impact of transcranial focused ultrasound on cortical activation in primates. Elife. (2019) 8:28. doi: 10.7554/eLife.40541

9. Hameroff S, Trakas M, Duffield C, Annabi E, Gerace MB, Boyle $\mathrm{P}$, et al. Transcranial ultrasound (TUS) effects on mental states: a pilot study. Brain Stimul. (2013) 6:409-15. doi: 10.1016/j.brs.2012. 05.002

10. Legon W, Sato TF, Opitz A, Mueller J, Barbour A, Williams A, et al. Transcranial focused ultrasound modulates the activity of primary somatosensory cortex in humans. Nat Neurosci. (2014) 17:322-9. doi: $10.1038 / \mathrm{nn} .3620$

11. Monti MM, Schnakers C, Korb AS, Bystritsky A, Vespa PM. Noninvasive ultrasonic thalamic stimulation in disorders of consciousness after severe brain injury: a first-in-man report. Brain Stimul. (2016) 9:940-1. doi: 10.1016/j.brs.2016.07.008

12. Nct02522429. Thalamic Low Intensity Focused Ultrasound in Acute Brain Injury (LIFUP). (2015). Available online at: https://clinicaltrials. gov/ct2/show/NCT02522429 (accessed February 18, 2019).

13. Bailey NW, Lewis PM, Thomson RH, Maller JJ, Junor P, Fitzgerald PB. Does exposure to diagnostic ultrasound modulate human nerve responses to magnetic stimulation? Ultrasound Med Biol. (2016) 42:2950-6. doi: 10.1016/j.ultrasmedbio.2016.08.003

14. Fini M, Tyler WJ. Transcranial focused ultrasound: a new tool for non-invasive neuromodulation. Int Rev Psychiatry. (2017) 29:168-77. doi: 10.1080/09540261.2017.1302924

15. Fomenko A, Neudorfer C, Dallapiazza RF, Kalia SK, Lozano AM. Lowintensity ultrasound neuromodulation: an overview of mechanisms and emerging human applications. Brain Stim. (2018) 11:1209-17. doi: 10.1016/j.brs.2018.08.013

16. Munoz F, Aurup C, Konofagou EE, Ferrera VP. Modulation of brain function and behavior by focused ultrasound. Curr Behav Neurosci Rep. (2018) 5:15364. doi: 10.1007/s40473-018-0156-7

17. Tyler WJ, Lani SW, Hwang GM. Ultrasonic modulation of neural circuit activity. Curr Opinion Neurobiol. (2018) 50:222-31. doi: 10.1016/j.conb.2018.04.011

18. Darrow DP. Focused ultrasound for neuromodulation. Neurotherapeutics. (2019) 16:88-99. doi: 10.1007/s13311-018-00691-3

19. Clement G, Hynynen K. A non-invasive method for focusing ultrasound through the human skull. Phys Med Biol. (2002) 47:1219. doi: 10.1088/0031-9155/47/8/301

20. Aubry J-F, Tanter M, Pernot M, Thomas J-L, Fink M. Experimental demonstration of noninvasive transskull adaptive focusing based on prior computed tomography scans. J Acous Soc Am. (2003) 113:84-93. doi: $10.1121 / 1.1529663$

21. Marquet F, Pernot M, Aubry J, Montaldo G, Marsac L, Tanter M, et al. Non-invasive transcranial ultrasound therapy based on a 3D CT scan: protocol validation and in vitro results. Phys Med Biol. (2009) 54:2597. doi: 10.1088/0031-9155/54/9/001

22. Marsac L, Chauvet D, Larrat B, Pernot M, Robert B, Fink M, et al. MR-guided adaptive focusing of therapeutic ultrasound beams in the human head. Med Phys. (2012) 39:1141-9. doi: 10.1118/1.3678988

23. Sukharev S, Corey DP. Mechanosensitive channels: multiplicity of families and gating paradigms. sci sTKE. (2004) 2004:re4. doi: 10.1126/stke.2192 004re4
24. Morris CE, Juranka PF. Lipid stress at play: mechanosensitivity of voltagegated channels. In: Current Topics in Membranes. Elsevier (2007a) p. 297-338. doi: 10.1016/S1063-5823(06)59011-8

25. Tyler WJ, Tufail Y, Finsterwald M, Tauchmann ML, Olson EJ, Majestic C. Remote excitation of neuronal circuits using low-intensity, low-frequency ultrasound. PLoS ONE. (2008) 3:e3511. doi: 10.1371/journal.pone.0003511

26. Morris CE, Juranka PF. Nav channel mechanosensitivity: activation and inactivation accelerate reversibly with stretch. Biophys J. (2007b) 93:822-33. doi: 10.1529/biophysj.106.101246

27. Plaksin $\mathrm{M}$, Shoham S, Kimmel E. Intramembrane cavitation as a predictive bio-piezoelectric mechanism for ultrasonic brain stimulation. Phys Rev. X. (2014) 4:011004. doi: 10.1103/PhysRevX.4.011004

28. Krasovitski B, Frenkel V, Shoham S, Kimmel E. Intramembrane cavitation as a unifying mechanism for ultrasound-induced bioeffects. Proc Natl Acad Sci. (2011) 108:3258-63. doi: 10.1073/pnas.1015771108

29. Kim H, Taghados SJ, Fischer K, Maeng LS, Park S, Yoo SS. Non-invasive transcranial stimulation of rat abducens nerve by focused ultrasound. Ultrasound Med Biol. (2012) 38:1568-75. doi: 10.1016/j.ultrasmedbio.2012.04.023

30. King RL, Brown JR, Newsome WT, Pauly KB. Effective parameters for ultrasound-induced in vivo neurostimulation. Ultrasound Med Biol. (2013) 39:312-31. doi: 10.1016/j.ultrasmedbio.2012.09.009

31. Menz MD, Oralkan O, Khuri-Yakub PT, Baccus SA. Precise neural stimulation in the retina using focused ultrasound. J Neurosci. (2013) 33:4550-60. doi: 10.1523/JNEUROSCI.3521-12.2013

32. Di Lazzaro V, Rothwell JC. Corticospinal activity evoked and modulated by non-invasive stimulation of the intact human motor cortex. J Physiol. (2014) 592:4115-28. doi: 10.1113/jphysiol.2014.274316

33. Ai L, Bansal P, Mueller JK, Legon W. Effects of transcranial focused ultrasound on human primary motor cortex using 7T fMRI: a pilot study. BMC Neurosci. (2018) 19:56. doi: 10.1186/s12868-018-0456-6

34. Legon W, Bansal P, Tyshynsky R, Ai L, Mueller JK. Transcranial focused ultrasound neuromodulation of the human primary motor cortex. Sci Rep. (2018) 8:10007. doi: 10.1038/s41598-018-28320-1

35. Legon W, Ai L, Bansal P, Mueller JK. Neuromodulation with single-element transcranial focused ultrasound in human thalamus. Hum Brain Mapp. (2018) 39:1995-2006. doi: 10.1002/hbm.23981

36. Leo A, Mueller JK, Grant A, Eryaman Y, Wynn L. Transcranial focused ultrasound for BOLD fMRI signal modulation in humans. Conf Proc IEEE Eng Med Biol Soc. (2016) 2016:1758-61. doi: 10.1109/EMBC.2016.7591057

37. Lee W, Kim HC, Jung Y, Chung YA, Song IU, Lee JH, et al. Transcranial focused ultrasound stimulation of human primary visual cortex. Sci Rep. (2016) 6:34026. doi: 10.1038/srep34026

38. Lee W, Kim H, Jung Y, Song IU, Chung YA, Yoo SS. Image-guided transcranial focused ultrasound stimulates human primary somatosensory cortex. Sci Rep. (2015) 5:8743. doi: 10.1038/srep08743

39. Mueller J, Legon W, Opitz A, Sato TF, Tyler WJ. Transcranial focused ultrasound modulates intrinsic and evoked EEG dynamics. Brain Stimul. (2014) 7:900-8. doi: 10.1016/j.brs.2014.08.008

40. Gibson BC, Sanguinetti JL, Badran BW, Yu AB, Klein EP, Abbott $\mathrm{CC}$, et al. Increased excitability induced in the primary motor cortex by transcranial ultrasound stimulation. Front Neurol. (2018) 9:1007. doi: 10.3389/fneur.2018.01007

41. Kim H, Chiu A, Lee SD, Fischer K, Yoo S-S. Focused ultrasound-mediated non-invasive brain stimulation: examination of sonication parameters. Brain Stimul. (2014) 7:748-56. doi: 10.1016/j.brs.2014.06.011

42. Lee W, Lee SD, Park MY, Foley L, Purcell-Estabrook E, Kim H, et al. Image-guided focused ultrasound-mediated regional brain stimulation in sheep. Ultrasound Med Biol. (2016) 42:459-70. doi: 10.1016/j.ultrasmedbio.2015.10.001

43. Fda. Marketing Clearance of Diagnostic 661 Ultrasound Systems and Transducers. Draft Guidance for Industry and Food and Drug 662 Administration Staff 2017. (2017). Available online at: https://www.fda. gov/MedicalDevices/DeviceRegulationandGuidance/GuidanceDocuments/ UCM578118 (accessed February 18, 2019).

44. Marquet F, Tung Y-S, Teichert T, Ferrera VP, Konofagou EE. Non-invasive, transient and selective blood-brain barrier opening in non-human primates in vivo. PLoS ONE. (2011) 6:e22598. doi: 10.1371/journal.pone.0022598 
45. Tsai H-C, Tsai C-H, Chen W-S, Inserra C, Wei K-C, Liu H-L. Safety evaluation of frequent application of microbubble-enhanced focused ultrasound blood-brain-barrier opening. Sci Rep. (2018) 8:17720. doi: 10.1038/s41598-018-35677-w

46. Lipsman N, Meng Y, Bethune AJ, Huang Y, Lam B, Masellis M, et al. Blood-brain barrier opening in Alzheimer's disease using MR-guided focused ultrasound. Nat Commun. (2018) 9:2336. doi: 10.1038/s41467-018-04529-6

47. Wang S, Olumolade OO, Sun T, Samiotaki G, Konofagou EE. Non-invasive, neuron-specific gene therapy can be facilitated by focused ultrasound and recombinant adeno-associated virus. Gene Ther. (2015) 22:104-10. doi: $10.1038 /$ gt.2014.91

48. Wang S, Kugelman T, Buch A, Herman M, Han Y, Karakatsani ME, et al. Non-invasive, focused ultrasound-facilitated gene delivery for optogenetics. Sci Rep. (2017) 7:39955. doi: 10.1038/srep39955

49. Thanou M, Gedroyc W. MRI-guided focused ultrasound as a new method of drug delivery. J Drug Deliv. (2013) 2013:616197. doi: 10.1155/2013/616197

50. Lee W, Chung YA, Jung Y, Song IU, Yoo SS. Simultaneous acoustic stimulation of human primary and secondary somatosensory cortices using transcranial focused ultrasound. BMC Neurosci. (2016) 17:68. doi: 10.1186/s12868-016-0303-6

51. Elias WJ, Lipsman N, Ondo WG, Ghanouni P, Kim YG, Lee W, et al. A randomized trial of focused ultrasound thalamotomy for essential tremor. $N$ Engl J Med. (2016) 375:730-9. doi: 10.1056/NEJMoa1600159

52. Meng Y, Huang Y, Solomon B, Hynynen K, Scantlebury N, Schwartz ML, et al. MRI-guided focused ultrasound thalamotomy for patients with medicallyrefractory essential tremor. J Vis Exp. (2017) 13:e56365. doi: 10.3791/56365

53. Meng Y, Solomon B, Boutet A, Llinas M, Scantlebury N, Huang Y, et al. Magnetic resonance-guided focused ultrasound thalamotomy for treatment of essential tremor: A 2-year outcome study. Mov Disord. (2018) 33:1647-50. doi: $10.1002 / \mathrm{mds} .99$

54. Park Y-S, Jung NY, Na YC, Chang JW. Four-year follow-up results of magnetic resonance-guided focused ultrasound thalamotomy for essential tremor. Mov Disord. (2019) 34:727-34. doi: 10.1002/mds.27637

55. Fasano A, De Vloo P, Llinas M, Hlasny E, Kucharczyk W, Hamani C, et al. Magnetic resonance imaging-guided focused ultrasound thalamotomy in parkinson tremor: reoperation after benefit decay. Mov Disord. (2018) 33:848-9. doi: 10.1002/mds.27348

56. Martínez-Fernández R, Rodríguez-Rojas R, Del Álamo M, HernándezFernández F, Pineda-Pardo JA, Dileone M, et al. Focused ultrasound subthalamotomy in patients with asymmetric Parkinson's disease: a pilot study. Lancet Neurol. (2018) 17:54-63. doi: 10.1016/S1474-4422(17)30403-9

57. Meng Y, Voisin MR, Suppiah S, Kalia SK, Kalia LV, Hamani C, et al. Is there a role for MR-guided focused ultrasound in Parkinson's disease? Mov Disord. (2018) 33:575-9. doi: 10.1002/mds.27308

58. Kim M, Kim C-H, Jung HH, Kim SJ, Chang JW. Treatment of major depressive disorder via magnetic resonance-guidedfocused ultrasound surgery. Biol Psychiatry. (2018) 83:e17-8. doi: 10.1016/j.biopsych.2017. 05.008

59. Mooney SJ, Nobrega JN, Levitt AJ, Hynynen K. Antidepressant effects of focused ultrasound induced blood-brain-barrier opening. Behav Brain Res. (2018) 342:57-61. doi: 10.1016/j.bbr.2018.01.004

60. Kim SJ, Roh D, Jung HH, Chang WS, Kim C-H, Chang JW. A study of novel bilateral thermal capsulotomy with focused ultrasound for treatmentrefractory obsessive-compulsive disorder: 2-year follow-up. J Psychiatry Neurosci. (2018) 43:327-37. doi: 10.1503/jpn.170188

61. Kumar KK, Bhati MT, Ravikumar VK, Ghanouni P, Stein SC, Halpern $\mathrm{CH}$. MR-guided focused ultrasound versus radiofrequency capsulotomy for treatment-refractory obsessive-compulsive disorder: a cost-effectiveness threshold analysis. Front Neurosci. (2019) 13:66. doi: 10.3389/fnins.2019. 00066

62. Jeanmonod D, Werner B, Morel A, Michels L, Zadicario E, Schiff G, et al. Transcranial magnetic resonance imaging-guided focused ultrasound: noninvasive central lateral thalamotomy for chronic neuropathic pain. Neurosurg Focus. (2012) 32:E1. doi: 10.3171/2011.10.FOCUS11248

63. Prabhala T, Hellman A, Walling I, Maietta T, Qian J, Burdette C, et al. External focused ultrasound treatment for neuropathic pain induced by common peroneal nerve injury. Neurosci Lett. (2018) 684:145-51. doi: 10.1016/j.neulet.2018.07.037

64. Yulug B, Hanoglu L, Kilic E. The neuroprotective effect of focused ultrasound: new perspectives on an old tool. Brain Res Bull. (2017) 131:199-206. doi: 10.1016/j.brainresbull.2017.04.015

65. Eames MD, Hananel A, Snell JW, Kassell NF, Aubry J-F. Trans-cranial focused ultrasound without hair shaving: feasibility study in an ex vivo cadaver model. J Therap Ultrasound. (2014) 1:24. doi: 10.1186/2050-5736-1-24

66. Guo H, Hamilton M, Offutt SJ, Gloeckner CD, Li T, Kim Y, et al. (2018). Ultrasound produces extensive brain activation via a cochlear pathway. Neuron. 98:1020-30.e1024. doi: 10.1016/j.neuron.2018.04.036

67. Sato T, Shapiro MG, Tsao DY. Ultrasonic neuromodulation causes widespread cortical activation via an indirect auditory mechanism. Neuron. (2018) 98:1031-41.e1035. doi: 10.1016/j.neuron.2018.05.009

Conflict of Interest Statement: The authors declare that the research was conducted in the absence of any commercial or financial relationships that could be construed as a potential conflict of interest.

Copyright (c) 2019 di Biase, Falato and Di Lazzaro. This is an open-access article distributed under the terms of the Creative Commons Attribution License (CC BY). The use, distribution or reproduction in other forums is permitted, provided the original author(s) and the copyright owner(s) are credited and that the original publication in this journal is cited, in accordance with accepted academic practice. No use, distribution or reproduction is permitted which does not comply with these terms. 